\title{
Surface-modified yeast cells: a novel eukaryotic carrier for oral application
}

Elisabeth E. Kenngott ${ }^{1 \#}$, Ruth Kiefer ${ }^{1}$, Nicole Schneider-Daum², Alf Hamann³ ${ }^{3}$ Marc Schneider ${ }^{4}$, Manfred J. Schmitt ${ }^{1}$, and Frank Breinig ${ }^{1 *}$

${ }^{1}$ Molecular and Cell Biology, Saarland University, D-66123 Saarbrücken, Germany; ${ }^{2}$ Helmholtz-Institute for Pharmaceutical Research Saarland, Department of Drug Delivery, Saarland University, D-66123 Saarbrücken, Germany; ${ }^{3}$ Charité Universitätsmedizin Berlin and Deutsches Rheuma-Forschungszentrum, D-10117 Berlin, Germany; ${ }^{4}$ Department of Pharmacy, Biopharmaceutics and Pharmaceutical Technology, Saarland University, D-66123 Saarbrücken, Germany.

\section{"Correspondence:}

Dr. Frank Breinig

Molecular and Cell Biology, Building A1.5, Saarland University, D-66123 Saarbrücken, Germany [Phone: (+49) 681 302-2211; Fax: (+49) 681 302-4710; E-Mail: fb@microbiol.uni-sb.de]

\#Present address: "Charité Universitätsmedizin Berlin and Deutsches RheumaForschungszentrum, D-10117 Berlin, Germany. 


\section{Abstract}

The effective targeting and subsequent binding of particulate carriers to $M$ cells in Peyer's patches of the gut is a prerequisite for the development of oral delivery systems. We have established a novel carrier system based on cell surface expression of the $\beta_{1}$-integrin binding domain of invasins derived from Yersinia enterocolitica and Y. pseudotuberculosis on the yeast Saccharomyces cerevisiae. All invasin derivatives were shown to be effectively expressed on the cell surface and recombinant yeast cells showed improved binding to both human HEp-2 cells and M-like cells in vitro. Among the different derivatives tested, the integrin-binding domain of $Y$. enterocolitica invasin proved to be the most effective and was able to target Peyer's patches in vivo. In conclusion, cell surface-modified yeasts might provide a novel bioadhesive, eukaryotic carrier system for efficient and targeted delivery of either antigens or drugs via the oral route.

\section{Keywords}

Oral delivery; Saccharomyces cerevisiae; cell surface-expression; invasin; M cell 


\section{Introduction}

Vaccination is one of the most successful and important means of modern medicine leading to a significant decrease of mortality caused by a number of infectious microbes in the last decades. However, due to an increasing resistance of known pathogenic microorganisms as well as the appearence of new pathogens, there is still a need for the development of novel vaccines. Most human pathogens like bacteria and viruses enter the body via the mucosal surfaces of the gastrointestinal and respiratory tracts. Therefore, newly developed vaccines should be able, beyond the induction of a protective cellular and humoral immune response, to provoke also a mucosa-associated immune response resulting in the production of IgA by the mucosal surfaces. The immunologically active sites in the gastrointestinal mucosa are called gut-associated lymphoid tissue (GALT), and contain organized lymphoid follicles (Peyer's patches, PP) [1]. A common feature of PP includes an epithelial surface containing $\mathrm{M}$ cells which are involved in luminal uptake, transport, and processing of mucosally introduced antigens. Following the uptake via $\mathrm{M}$ cells, the particular antigen is transported to immune cells such as $T$ and B lymphocytes, dendritic cells, and macrophages which are located beneath the surface of the PP in order to finally induce an effective immune response [2, 3]. Characteristically, M cell-mediated antigen uptake is associated with the development of an secretoric $\lg \mathrm{A}(\mathrm{s} \lg \mathrm{A})$ response [4]. A strong mucosal immune response is able to prevent the infectious agent from attaching and colonising the mucosal epithelium (non-invasive bacteria) or from penetrating and replicating in the mucosa (viruses and invasive bacteria). It can also block microbial toxins from binding to and affecting epithelial and other target cells.

However, only a few of today's vaccines are applied via the mucosal route. Most of the currently available vaccines are licensed for use only via a nonmucosal route, usually subcutaneous or intramuscular inoculation [5]. One of the most critical factors for the 
induction of protective mucosal immune responses represents the effective adhesion of the antigens to the mucosal surface and, in particular, the embedded M cells [2]. Some pathogenic organisms, including Yersinia, have developed a mechanism to specifically exploit $M$ cells in order to invade the body. These pathogens express specialized surface proteins, so called invasins, that can bind to surface receptors on mammalian cells and lead to an enhanced uptake of Yersinia by these cells [6, 7]. The invasin from Yersinia pseudotuberculosis represents a 986 amino acid protein of the outer membrane [8]. The carboxy terminal 192 amino acids of this protein bind to some of the members of the $\beta_{1}$-integrin superfamily, while the remainder of the protein is responsible for the presentation of the binding domain on the cell surface $[9,10] . Y$. enterocolitica possesses a homologue invasin but both proteins are not identical [11]. With respect to the integrin receptors, it shows the same binding behavior as pseudotuberculosis invasin [12]. Similar to the invasin of Y. pseudotuberculosis, the integrin-binding domain is located in the C-terminal region of the protein [13]. In line with this, Critchley-Thorne et al. (2006) showed in a mouse model that expression of invasin from Y. pseudotuberculosis on non-pathogenic Escherichia coli cells leads to uptake of these bacteria by M cells in the Peyer's patches [14]. Likewise, expression of $Y$. pseudotuberculosis invasin on the surface of Salmonella typhimurium resulted in increased translocation of the recombinant bacteria from the intestinal lumen into the mesenteric lymph nodes [15]. Further, invasin-coated particles were successfully used for targeting to different cell lines, suggesting a high potential of such adhesins with regard to the development of novel, so called bioadhesive carriers [16].

In the last years, we and other groups described the successful usage of intact, recombinant yeast cells for the delivery of both proteins [17-22] and functional nucleic acids [23-28] indicating the potential of yeast cells for the development of novel vaccines. Here, we report the construction of a novel, bioadhesive delivery system 
based on the surface-expression of the integrin binding domains of $Y$. enterocolitica or Y. pseudotuberculosis invasin on the baker's yeast Saccharomyces cerevisiae. The recombinant yeast cells showed a significant binding to human epithelial cells and $M$ cells in vitro as well as Peyer's patches in vivo, rising the possibility to use the surfacemodified yeast cells as carrier for efficient oral delivery of either antigens or drugs in the human gut. 


\section{Materials and methods}

Strains, culture media and methods

Standard molecular manipulations were performed as described [29]. For cloning, E. coli strain TOP10 (F-mcrA $\Delta(m r r-h s d R M S-m r c B C)$ \$80lacZM15 $\Delta$ lacX74 recA1 deoR araD139 $\Delta\left(\right.$ ara-leu)7697 gall ga/K rspL (Str $\left.{ }^{\mathrm{R}}\right)$ endA1 nupG) was grown at $37^{\circ} \mathrm{C}$ in LB-medium supplemented with $100 \mu \mathrm{g}$ ampicillin $\mathrm{ml}^{-1}$ when necessary. For the in vivo expression of all constructed fusion proteins, S. cerevisiae strain EBY100 (MATa ura 3-52 trp 1 leu2 $\Delta 1$ his3 200 pep4:HIS3 prb1 $\Delta 1.6 \mathrm{R}$ can1 GAL; strain includes $A G A 1$ gene under control of a GAL promotor and URA3 as selection marker; Invitrogen) was used. Cells were grown in YEPD medium at $30^{\circ} \mathrm{C}$. Transformation was carried out by the lithium acetate method according to Ito et al. [30] and transformants were selected on trp $\mathrm{d} / \mathrm{o}$ medium. To express the fusion proteins, cells were grown in YNB-CAA medium containing glucose overnight and were then switched to galactose-containing YNB-CAA. PCR amplifications were performed using high fidelity Taq DNApolymerase (Roche) according to the instructions of the manufacturer.

\section{Construction of cell wall fusion proteins}

The genes encoding the extracellular part of invasin of $Y$. enterocolitica or $Y$. pseudotuberculosis were optimised for codon usage of $S$. cerevisiae by the synthesis of an artificial gene sequence (GeneArt, Regensburg) encoding the Cterminal 397 amino acids (aa; Y. enterocolitica) and 497 aa (Y. pseudotuberculosis) of the natural invasin protein. For cloning, EcoRI and Notl restriction sites were introduced. The shortened invasin variants (198 aa in case of $Y$. enterocolitica and 197 aa for Y.pseudotuberculosis, respectively) were PCR-amplified using the particular artificial gene sequence as template and the following primers: 5' Y.entınv BD EcoRI (5'-GAATTCATCACCTTGTACCCAATTCCAAAGATTACCTC-3'), 3' Y.entinv BD Notl 
(5'-GCGGCCGCTCATCATTGTGG-3'), 5' $\quad$ Y.pstınv BD EcoRI (5'GAATTCATTTCCTTGTTCCCAGTTCCAACTTTGACC-3') and 3' Y.pstınv BD Notl (5'GCGGCCGCTCATCAGATGGAC-3'). Invasin derivatives were then inserted in the pYD1 expression vector (Invitrogen) via EcoRI/Notl restriction, yielding the plasmids pYD1-Y.ent397, pYD1-Y.pst497, pYD1-Y.ent198, and pYD1-Y.pst197 (see Fig.1).

\section{Indirect immunofluorescence}

Indirect immunofluorescence was performed with a monoclonal Anti-Xpress Antibody (Invitrogen) according to the manufacturer specifications. In brief, exponentially growing yeast cells were harvested, washed with PBS $(\mathrm{pH} 7.4)$ and incubated with anti-Xpress antibody diluted $1 / 100$ in PBS for one hour at room temperature with occasional mixing. Subsequently, the cells were washed three times with PBS and incubated with a fluoresceine isothiocyanate (FITC)-labeled anti-mouse-lgG (Roche) for one hour at room temperature in the dark. After washing, cells were examined on a fluorescence microscope (Biozerø; KEYENCE) using $488 \mathrm{~nm}$ as excitation wavelength.

\section{Cell culture}

The human adherent epithelial cell lines HEp-2 and Caco-2 were cultured in Dulbecco's modified Eagle medium (DMEM; Sigma) supplemented with $10 \%$ fetal calf serum (FCS; Biochrom), penicillin (50 $\left.\mathrm{U} \mathrm{ml}^{-1}\right)$ and streptomycin (50 $\mathrm{g} \mathrm{m} \mathrm{m}^{-1}$; Biochrom). The suspension cell line Raji was cultured in RPMI 1640 (Sigma) supplemented with $10 \%$ fetal calf serum (FCS), penicillin $\left(50 \mathrm{U} \mathrm{ml}^{-1}\right)$ and streptomycin $\left(50 \mu \mathrm{g} \mathrm{ml}^{-1}\right)$. Cells were grown in a humidified $5 \% \mathrm{CO}_{2}$ atmosphere at $37^{\circ} \mathrm{C}$.

In vitro infection of HEp-2 monolayers with yeast cells 
Yeast cells induced in YNB-CAA medium containing galactose were harvested by centrifugation, washed three times with sterile PBS, counted and resuspended in cell culture medium. In preparation for either assay, HEp-2 cells were released with trypsin/EDTA (Sigma) for some minutes at $37^{\circ} \mathrm{C}$ and replaced into a 24 -well plate, and grown to nearly confluency. Subsequently, $5 \times 10^{6}$ yeast cells per well were added; after an incubation step of 3 hours at $37^{\circ} \mathrm{C}$ and $5 \% \mathrm{CO}_{2}$ the cultures were carefully washed several times with cell culture medium to remove unbound yeast cells. Subsequently, human cells were lysed by the addition of $0.1 \%$ Triton X-100 and the remaining yeast cells were harvested by centrifugation. The number of bound yeast cells was enumerated by plating appropriate dilutions on YEPD agar and incubation at $30^{\circ} \mathrm{C}$.

\section{In vitro $M$ cell model}

M-like cells were generated as described [31, 32]. In brief, Caco-2 were seeded onto porous membranes in Transwell plates (Corning; $1.12 \mathrm{~cm}^{2}$, pore size: $3 \mu \mathrm{m}$ ) and incubated at $37^{\circ} \mathrm{C}$ and $5 \% \mathrm{CO}_{2}$ to a confluency of at least $80 \%$ in order to allow full differentiation before addition of $1.8 \times 10^{5}$ Raji $B$ cells in the basal chamber. The cocultures were incubated for further 5 days in a medium consisting of $1 / 3 \mathrm{RPMI}$ and $2 / 3$ DMEM. Morphological changes after co-culture were checked microscopically using Phalloidin-TRITC staining [32]. The Caco-2 monolayer was washed once with PBS and fixed with $3.7 \%$ formaldehyde for $30 \mathrm{~min}$. After two more washing steps, $1.8 \times 10^{6}$ yeast cells were added to the apical chamber in a volume of $500 \mu \mathrm{l}$ and the basal chamber was filled with $1.5 \mathrm{ml}$ fresh medium. Following an incubation of two hours at $37^{\circ} \mathrm{C}$ and $5 \% \mathrm{CO}_{2}$, the Caco-2 monolayer was carefully washed three times with PBS to eliminate unbound yeast cells. Eventually, the mammalian cells were lysed by 
adding $0.1 \%$ Triton $\mathrm{X}-100$ and the remaining yeast cells were plated in different dilutions on YEPD agar.

Assessment of cell monolayer integrity and morphological as well as functional changes in co-cultures

Cell monolayer integrity and morphological changes during co-culture were assessed by labeling F-actin with TRITC-Phalloidin (Life Technologies) and subsequent microscopical analysis. Monolayers were washed with HBSS buffer and fixed by incubation with $3.7 \%$ formaldehyde for 10 minutes. After two washing steps with HBSS, cells were first incubated for 10 minutes with $0.1 \%$ TritonX 100 (Sigma) at room temperature, washed again and incubated for 1 hour with TRITC-Phalloidin in PBS (1 $\mu \mathrm{g} / \mathrm{ml}$ ). After extensive washing, the filter membrane was removed, placed on a glass slide and immediately analyzed by laser scanning microscopy.

Cell monolayer integrity was analyzed by measurement of the Trans-Epithelial Electrical Resistance (TEER). Monolayers were gently washed with pre-warmed HBSS and measured at $37^{\circ} \mathrm{C}$ using an $\mathrm{Ag} / \mathrm{AgCl}$ electrode. TEER values are depicted in unit area resistance $\left(\Omega \mathrm{cm}^{2}\right)=$ resistance $(\Omega)$ x effective membrane area $\left(\mathrm{cm}^{2}\right)$.

To detect functional changes in mono- versus co-cultures, transport efficiency of monolayers was analyzed using fluorescent microspheres. Fluoresbrite ${ }^{\circledR}$ Microparticles $(0,46 \mu \mathrm{m}$; Polysciences, Inc.) diluted in cell culture medium were introduced into the apical chamber of transwells. The basolateral solution was sampled at several time points and analyzed using an ELISA reader. Percentage of translocation was calculated using the donor solution for calibration. After incubating the co-culture in the microsphere solution for 250 minutes, monolayers were analyzed microscopically as described earlier. 
In vivo visualisation of yeast cell uptake

For in vivo analysis of yeast cell uptake in intestinal mucosa, yeast cells were labeled with 5-(6)-Carboxyflourescein diacetate $N$-succinimidylester (CFSE; Sigma). For this purpose, lyophilized yeast cells were washed twice with PBS and resuspended in PBS containing $0.1 \%$ DMSO and $1 \mu \mathrm{M}$ CFSE. After 90 minutes incubation, the cells were washed three times with PBS to remove remaining dye. Female BALB/c mice were purchased from Charles River, housed in a specific pathogen free environment and used for experiments at 8-10 weeks of age. All experiments were approved by the Landesamt für Gesundheit und Soziales (LAGeSo; G 0014/14). Mice were treated orally by gavage with $1 \times 10^{8}$ CFSE labeled yeast cells (negative control) or with Y.ent 198 expressing yeast ( $n=3$ for each group) resuspended in $200 \mu$ I PBS. After four hours, mice were sacrificed by cervical dislocation and the small intestine was removed. A 2-3 cm segment from the jejunum containing at least one PP was isolated, fixed for 16 hours in PBS containing $2 \%$ PFA and dehydrated by incubation in solutions of increasing sucrose concentrations starting with $10 \%, 20 \%$ and finally $30 \%$, each for 8-16 hours at $4^{\circ} \mathrm{C}$. The tissue was embedded in Tissue-Tec O.T.C. cryo embedding medium (Sakura Finetec), frozen and $7 \mu \mathrm{m}$ sections were cut using a HM 560 Cryotom (Thermo Fischer Scientific). Sections were stored at $-20^{\circ} \mathrm{C}$.

Prior to confocal microscopy the sections were warmed up to room temperature and mounted with DAKO mounting medium containing DAPI (Sigma). Slides were analyzed with a LSM 710 confocal microscope (Zeiss) and pictures were taken using the software Zen 2011 (Zeiss). 


\section{Results}

Construction of invasin fusion proteins and targeting to the cell wall

In order to develop a yeast-based carrier for oral application we used the wellestablished Aga1/Aga2 system in which the protein of interest is fused to Aga2 and subsequently anchored via Aga1 to the yeast cell surface (Fig.1A; [33]). We constructed fusion proteins consisting of Aga2 and the invasins of Y. pseudotuberculosis (Y.pst) and Y. enterocolitica (Y.ent) in two different lengths corresponding to the complete extracellular invasin domains (Y.pst497 and Y.ent397) or the adhesion domains alone (Y.pst197 and Y.ent198), respectively (Fig. 1B; [34]). In order to ensure proper translation the invasin sequences were codon-optimized for yeast.

(A)

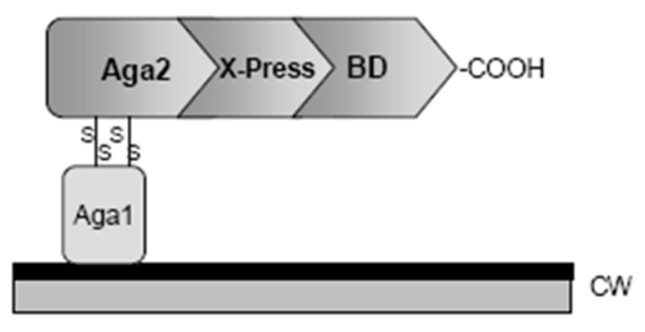

(B)

\begin{tabular}{l|l|l|l|}
\hline Aga2 & Xpress & Y.pst $_{197}$ & Y.pst $_{197}$ \\
\hline Aga2 & Xpress & Y.pst $_{497}$ & Y.pst $_{97}$ \\
\hline \multicolumn{2}{|l}{} \\
\hline Aga2 & Xpress & Y.ent $_{198}$ & Y.ent $_{198}$ \\
\hline Aga2 & Xpress & Y.ent $_{397}$ & Y.ent $_{397}$ \\
\hline
\end{tabular}

Figure 1: 


\section{(A) Schematical drawing showing the cellular anchoring of the integrin-binding}

domain of invasin in S. cerevisae. Cell wall-anchored Aga1 is covalently linked to a fusion protein consisting of Aga2, the X-press epitope and the respective invasinderivative; the integrin-binding domain is located near the C-terminus of the fusion protein. BD: integrin-binding domain; CW: cell wall. (B) Schematic representation of the different invasin derivatives used in this study. Y.pst: Y. pseudotuberculosis; Y.ent: $Y$. enterocolitica; the respective numbers indicate the length of the C-terminal domain of each invasin.

After GAL1-induced expression, localization of the respective cell wall fusion was investigated by indirect immunofluorescence probed with antibodies against the incorporated Xpress tag (as the correct invasin specific antibodies were not available in our lab). As expected, all yeast transformants showed a bright fluorescence that was localized over the entire cell wall whereas control cells showed no fluorescence (Fig. 2A). 


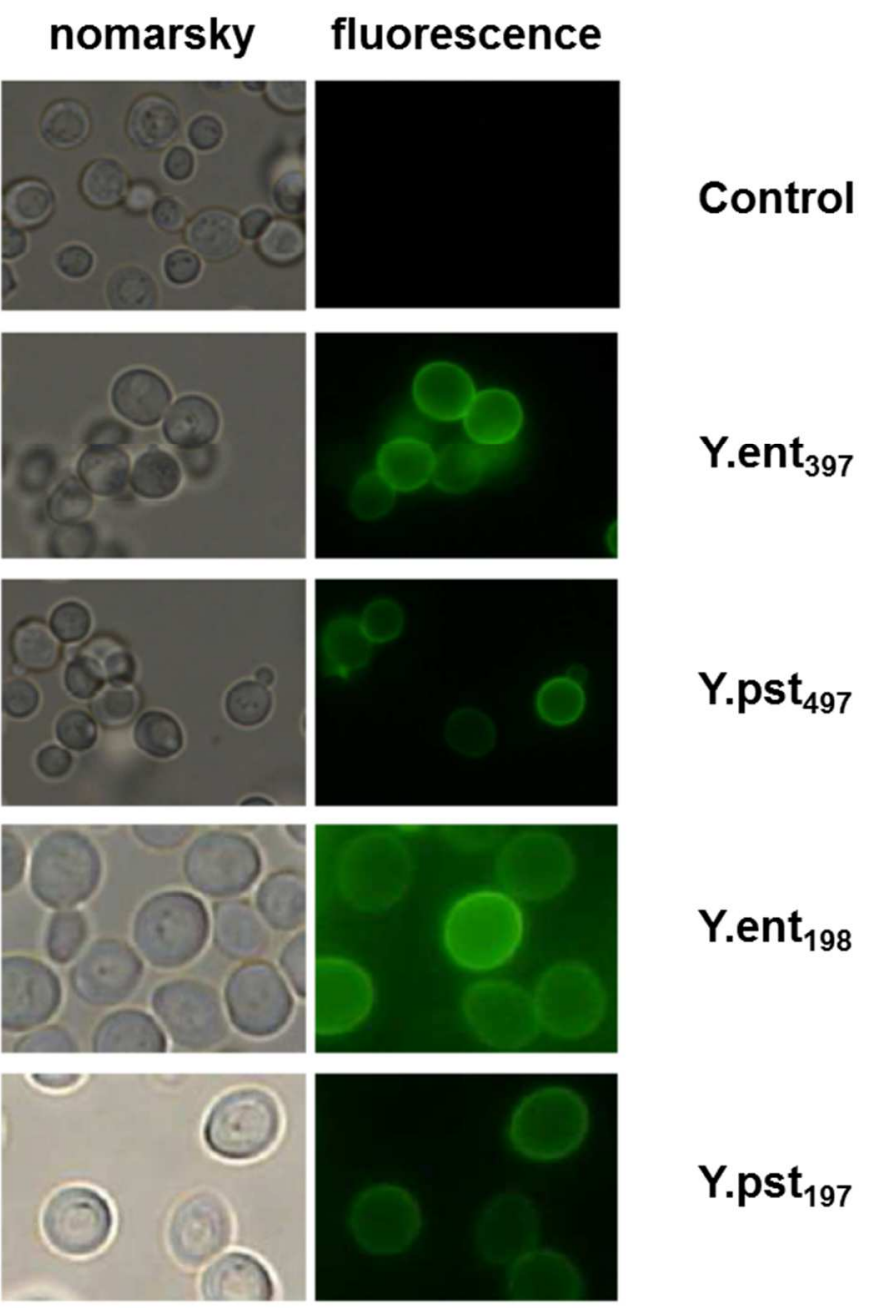

\section{Figure 2:}

The Aga2/invasin fusions are located at the yeast cell surface. S. cerevisiae strain EBY100 was transformed with the respective invasin derivative and expression of the cell wall fusion protein was investigated using indirect immunofluorescence. Exponential growing yeast cells were stained with a monoclonal anti-X-press-antibody followed by treatment with a FITC-labeled secondary antibody. A nomarsky image as well as the corresponding fluorescence image are shown.

However, as the Xpress tag is also present in the empty control vector, a simple detection of fluorescence is not a direct proof for a successful expression of the invasin 
fusions. Therefore, we performed a Western Blot with cell wall extracts after DTTtreatment yielding specific signals and demonstrating that the Aga2/invasin derivatives were successfully targeted to the yeast cell wall (not shown). Subsequent FACS analysis revealed that about $56 \%$ of the control cells (empty vector) expressed Aga2 whereas expression rates of the invasin derivatives were slightly lower with $35 \%$ (Y.ent397) and $39 \%$ (Y.ent 198$)$ for Y.ent, and $44 \%$ (Y.pst497) and $42 \%$ (Y.pst 197$)$ for Y.pst, respectively. This confirms results from Moelleken and Hegemann who expressed the adhesion domain Y. pseudotuberculosis invasin on S. cerevisiae using the same anchoring system [35]. Taken together, our results show that the different invasin derivatives can be successfully targeted in roughly equal amounts to the cell wall of $S$. cerevisiae.

Cell wall-anchored invasin derivatives differ in their ability to bind to human HEp-2 cells As invasin is known to interact with $\beta_{1}$-integrins on the surface of human cells [7] we tested whether the different invasin derivatives mediate a binding of the recombinant yeast cells to HEp-2 cells. To do so, we incubated yeast cells expressing the different invasin variants with HEp-2 cells seeded in well plates and determined the amount of bound yeast cells. In contrast to yeast cells expressing Aga2 alone, yeast cells presenting invasin on their surface showed a significant affinity for HEp-2 cells (Fig. 3; $\mathrm{p}<0.05$ for all invasin derivatives). In particular, although not entirely statistically significant ( $p=0.07$ ), Y.ent mediated a considerably higher binding to HEp-2 cells than Y.pst. Regarding the length of the invasin domains there was a significant higher binding of the shorter derivatives containing only the actual adhesion domain (Y.ent 198 and Y.pst $197 ; p<0.05$ each). Considering the fact that $Y . e n t$ is slightly less expressed than Y.pst (see above), these specific differences clearly argue for an invasin- 
dependent interaction of the surface-modified yeast cells with ligands on the surface of HEp-2 cells.

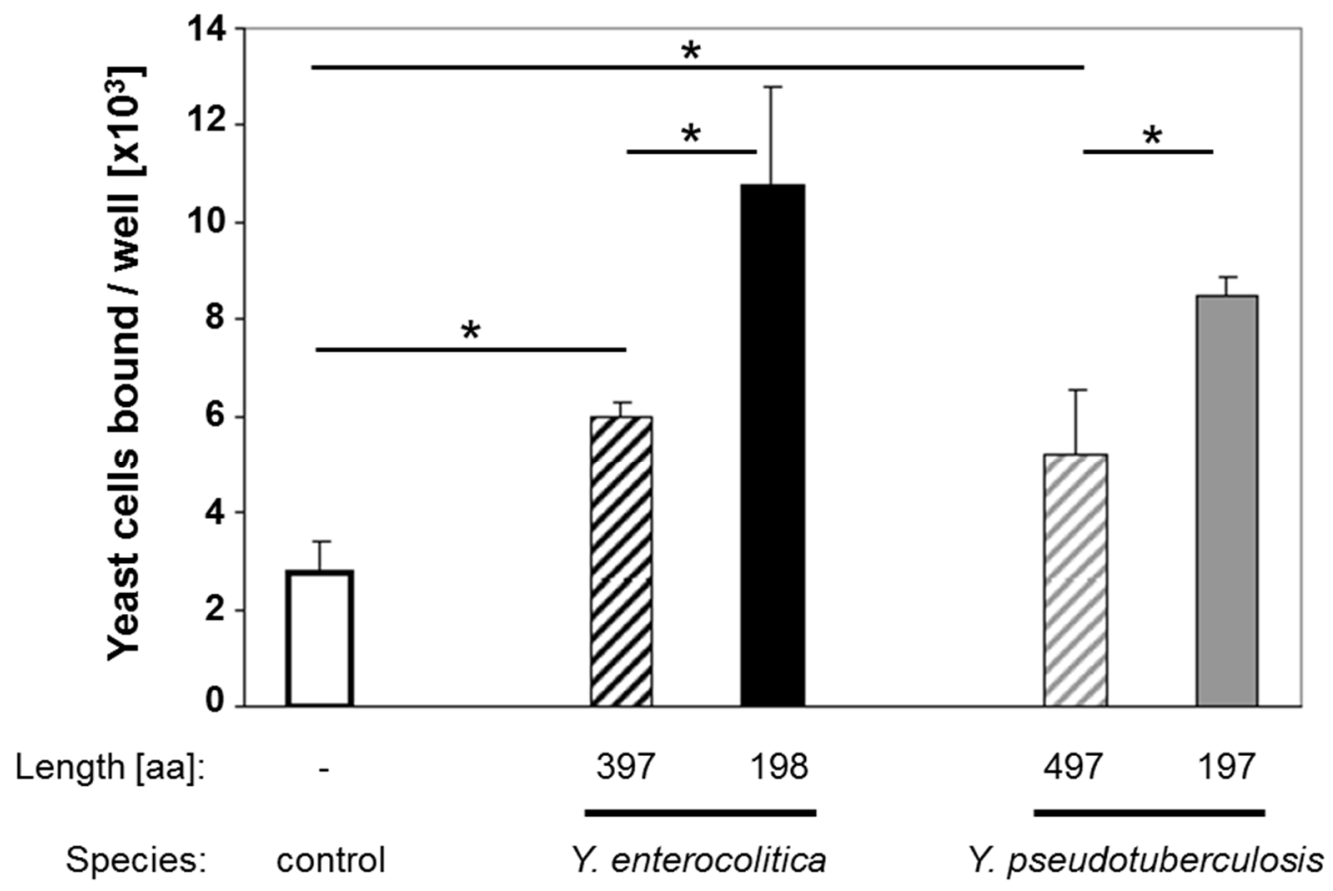

\section{Figure 3:}

Binding of surface-modified yeast cells to human HEp-2 cells. $5 \times 10^{6}$ yeast cells were added to $\mathrm{HEp}-2$ cells and incubated for 4 hours at $37^{\circ} \mathrm{C}$ and $5 \% \mathrm{CO}_{2}$ atmosphere. Subsequently, the cultures were washed several times with PBS and the HEp-2 cells were lysed with $1 \%$ Triton X-100. The yeast cells were washed with PBS and plated out on YEPD agar to enumerate the number of yeast cells bound. A vector containing only Aga2 without invasin domain was used as negative control. The standard deviation of 6 independent experiments is given $\left({ }^{*} p<0.05\right)$.

\section{Surface-modified yeast cells bind to M-like cells}

Adhesion of a particular carrier to $\mathrm{M}$ cells within the mucosal surfaces of the gut is one of the most promising strategies for the induction of mucosal immune responses [5]. Consequently, after having established the yeast-based display system for invasin, we 
next asked whether the recombinant yeast cells are also able to mediate a binding to in vitro generated $\mathrm{M}$-like cells. In order to investigate this we used a well-established system in which the co-culturing of human intestinal epithelial Caco-2 cells and Raji B cells leads to the differentiation of a subpopulation of epithelial cells into M-like cells (Fig. 4A; [31, 32]). For these experiments we used only the short invasin variants that showed the highest binding in the HEp-2 system (Y.ent198 and Y.pst197; see above). After incubation with the respective yeast transformants, the number of yeast cells bound to the co-cultured Caco-2/Raji B cells was determined and compared to the amount of cells bound to a standard Caco-2 monolayer (Fig. 4B). Morphological and functional changes resulting from the co-culture were routinely checked by measuring TEER-values, translocation efficiency of fluorescent microparticles, and also by Factin/Phalloidin staining (see suppl. Fig. 1). 
(A)

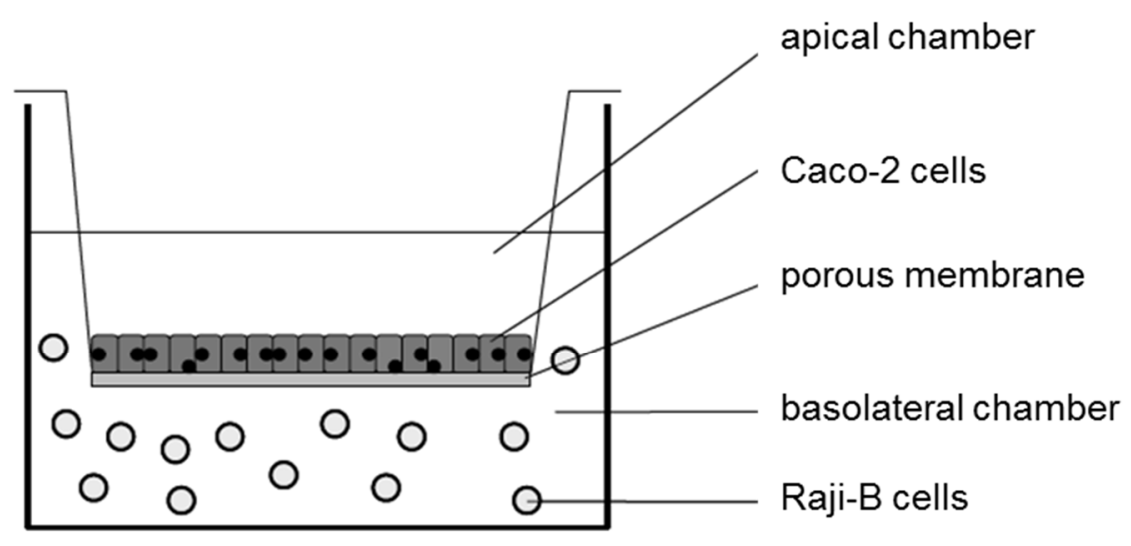

(B)

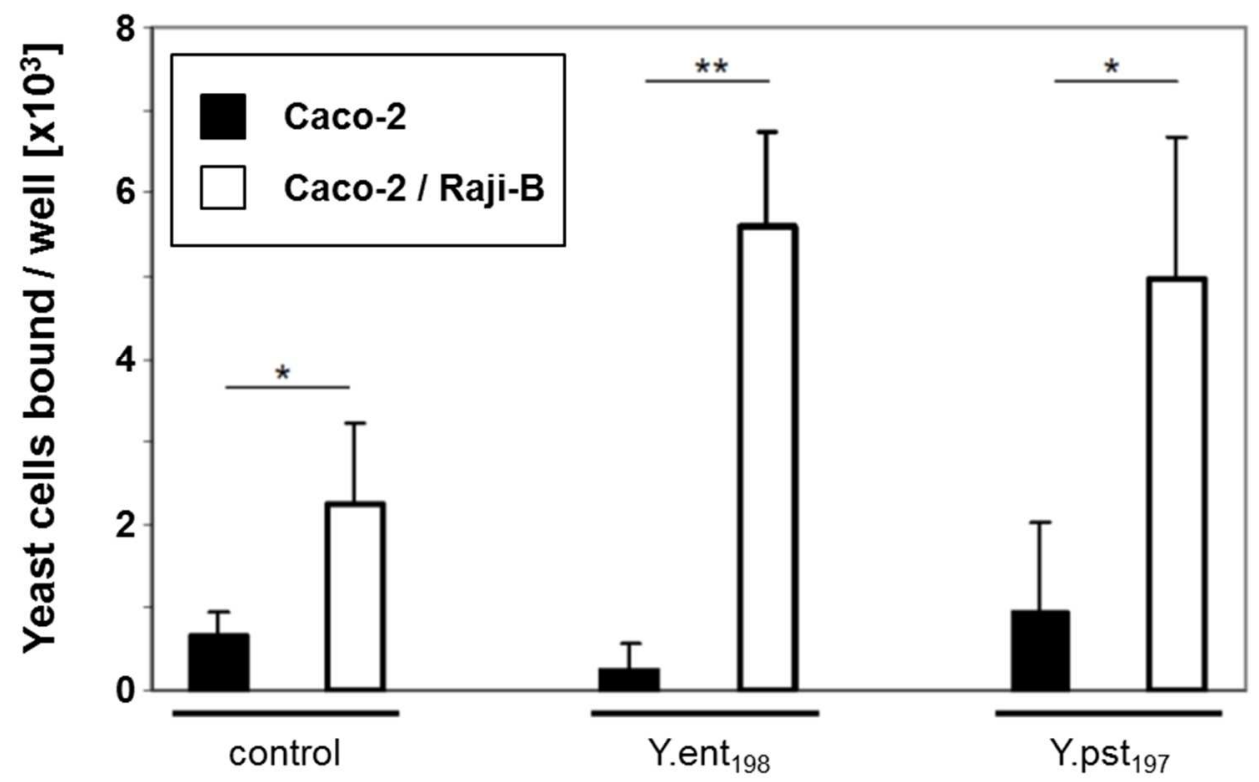

Figure 4:

(A) Schematical drawing of the in vitro $\mathrm{M}$ cell model used in this study. Caco-2 cells were seeded onto a porous membrane and cultivated to confluency. Subsequently, Raji B cells were added to the basolateral chamber; after another one week of incubation, a subpopulation of the Caco-2 cells differentiates into $M$ cells. (B) Binding of surface-modified yeast cells to in vitro-generated M-like cells. $5 \times 10^{6}$ yeast cells were added to either Caco-2 cells alone or a Caco-2/Raji B cell co-culture 
and incubated for 4 hours at $37^{\circ} \mathrm{C}$ and $5 \% \mathrm{CO}_{2}$ atmosphere. Subsequently, the cultures were washed several times with PBS and the number of yeast cells bound was determined after plating on YEPD agar. A vector containing only Aga2 without invasin domain was used as negative control. The standard deviation of 4 independent experiments is given $\left({ }^{*} p<0.05 ;{ }^{* *} p<0.01\right)$.

In particular, the invasin-expressing yeast cells showed a significant higher affinity to the co-cultured cells than to Caco-2 cells alone (Y.ent198: $p<0.01$; Y.pst197: $p<0.05$ ). Interestingly, also the binding of the control cells expressing Aga2 only was significantly increased $(p<0.05)$, clearly indicating that the co-culture of Caco-2 and Raji B cells leads to specific changes in the Caco-2 monolayer as orginally described for the in vitro $\mathrm{M}$ cell model $[31,32]$. However, both invasin-displaying yeast transformants showed a significant higher binding than the control cells ( $p<0.05$ each), suggesting that the binding is mediated by an interaction of the invasin adhesion domains to specific ligands at the surface of the human cells. Again, and in agreement with the data derived from the HEp-2 model (see above), expression of Y.ent proved to be more effective in comparison to the Y.pst invasin (Fig. 4). Taken together, these results clearly account for an invasin-dependent binding of the surface-modified yeast cells to human M-like cells.

Surface-expression of Y.ent 198 increases targeting to the small intestine in vivo To test whether surface modification of yeast actually leads to an increased uptake via the intestinal mucosa in vivo we fed mice with fluorecently labeled yeast cells expressing Y.ent 198 as this derivative yielded the best in vitro binding. Four hours after treatment, mice were sacrificed and the transport of yeast cells into the tissue of the 
jejunum was analyzed microscopically. In none of the analyzed samples yeast cell uptake within in the villous region of the small intestine was observed. In contrast, the CFSE-labeled yeast cells detected in these sections were restricted to the gut lumen (Figure 5, top row). As already shown in the literature, isolated signals were observed within PP of mice fed with control yeast (Figure 5, center) showing that yeast cells can principally be taken up into lymphoid structures in the small intestine [36]. However, the uptake in PP tissue was highly increased in animals treated with Y.ent198expressing yeast cells (Figure 5, bottom) with a scattering of the CFSE signal indicating a vesicular distribution within the PP cells confirming both a successful and effective targeting of the Y.ent 198 -expressing yeast cells to PP.

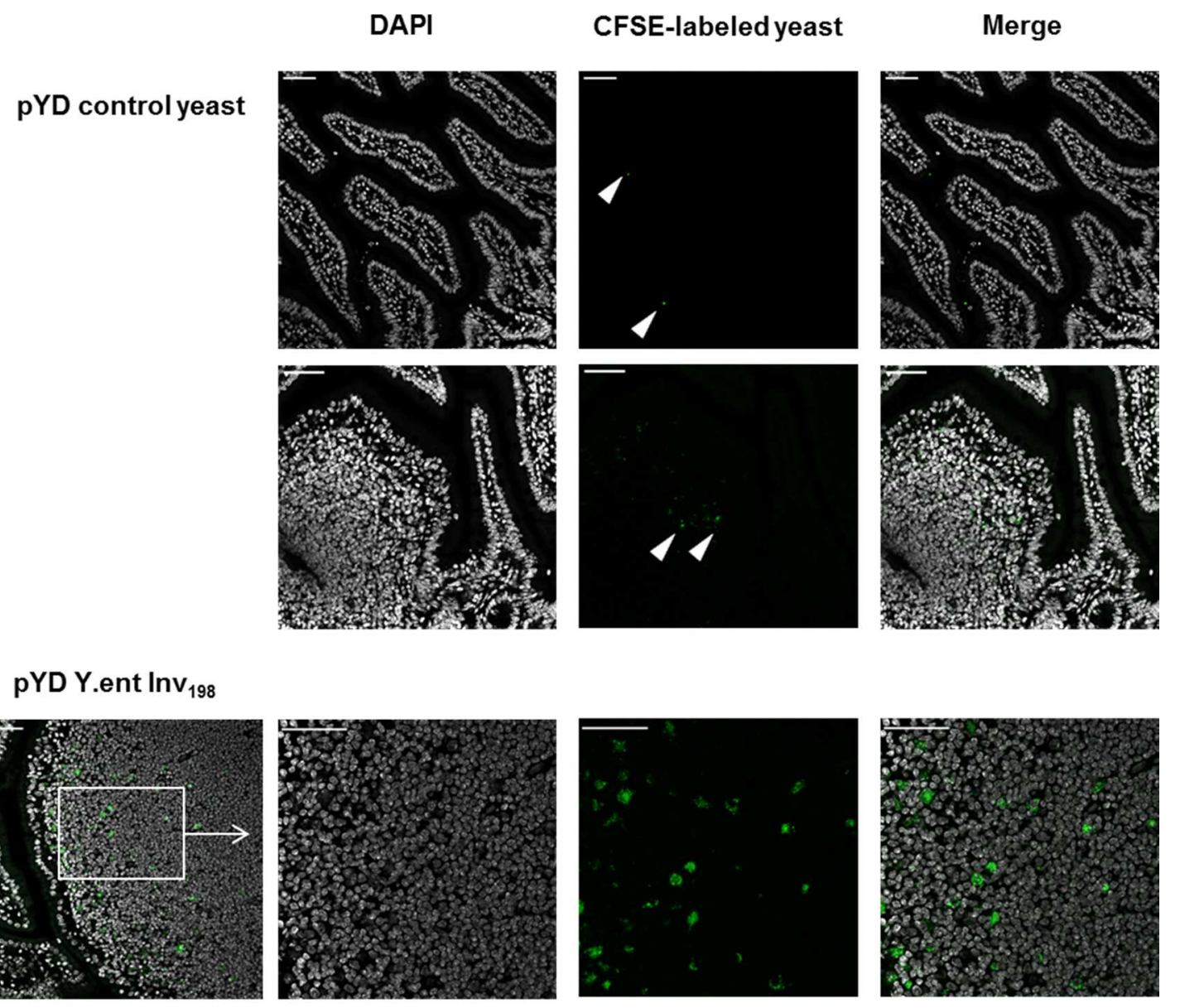

Figure 5: 
Invasin expression increases targeting to small intestinal Peyer's patches in vivo. $\mathrm{BALB} / \mathrm{c}$ mice were fed with $1 \times 10^{8}$ CFSE-labeled yeast cells expressing either Y.ent 198 or a vector containing only Aga2 without invasin domain as negative control by oral gavage. Four hours later, the mice were sacrificed, the small intestine removed, washed and prepared for subsequent cryosectioning. Sections were mounted with DAPI-containing mounting medium prior to analysis by confocal laser microscopy. Each sample represents one of three similar results ( $n=3$; scale bar: $50 \mu m$ ). 


\section{Discussion}

Despite the many attractive features of mucosal immunisation, it has often proven difficult in practice to stimulate protective mucosal immune responses. The main reason for this finding lies in the fact that orally applied vaccines encounter several host defence mechanisms such as epithelial barriers, mucus or a variety of proteolytic and lipolytic enzymes. Thus, in analogy to successful mucosal pathogens, a potential carrier should possess some key features: (i) it should be particulate, (ii) protect the delivered substance from proteolytic degradation in stomach and intestine (iii) adhere selectively to the $M$ cells within mucosal surfaces, (iv) efficiently stimulate innate immune responses, and (v) induce adaptive immune responses appropriate for the respective target pathogen/s [1, 37-39].

Yeast cells are naturally able to fulfil the qualifications for such a carrier in a unique manner: yeast per se is particulate with a size of a few micrometers, protects a foreign antigen from degradation during the stomach passage by acidic $\mathrm{pH}$ or by proteases in the gut and remains viable in the intestine for up to a week [40]. It is taken up by $M$ cells [36] and is known to possess adjuvant effects by stimulating innate immune responses by structural cell wall components [20,41, 42]. In particular, yeast is able to stimulate TLR2 and 4 which have been shown to enhance microparticle uptake by M cells and to support mucosal adjuvantic properties of a carrier [2, 43, 44]. Moreover, it has been shown to generate adaptive (including cell-mediated) immune responses in mice after subcutaneous or oral application [19, 45-47] and also in humans [48]. Importantly, the potential of yeast as novel life vaccine reaches far beyond the criteria mentioned above: in contrast to the majority of attenuated bacteria and mammalian viruses (which are already under investigation as mucosal vaccine candidates, e.g. Listeria) several yeast genera possess the GRAS (generally regarded as safe) status which should facilitate the approval of a yeast-based vaccine considerably. 
Additionally, yeast combines the advantages of a single-cell microorganism (small size, easy cultivation, rapid cell growth) with the advantages of a eukaryotic cell (ability to perform complex posttranslational modifications, lack of endotoxins). Further, many yeast genera are, due to the tremendous experience in using yeast in genetics and biotechnology for many years, also accessible to several genetical and structural modifications allowing amongst others the development of multivalent vaccines or the modulation of immune responses induced [20,21]. Eventually, almost essential for the usage in developing countries, they can be easily stored as dry-yeasts making a coldchain fully dispensable.

In order to enhance the natural features of a yeast-based carrier for a targeted delivery of antigens or drugs via $\mathrm{M}$ cells, we expressed different derivatives of invasins derived from Y. pseudotuberculosis and Y. enterocolitica on the cell surface of the baker's yeast S. cerevisiae using the well-established Aga1/Aga2 expression system [33]. In this system, the Aga2-invasin fusions are linked via disulfide-bonds to the cell wallanchored Aga1 moiety. Every invasin derivative was successfully targeted to the cell wall and could be released by cleavage of the disulfide-bond after DTT-treatment (not shown). All invasin-expressing yeast recombinants bound to human HEp-2 cells (Fig. 3) demonstrating that the invasin binding domain on their cell surface is fully functional. These results confirm data from Moelleken and Hegemann who expressed the binding domain of $Y$. pseudotuberculosis invasin in yeast using the same anchoring system and also found an enhanced binding of the surface-modified yeast cells to HEp-2 cells [35]. The shorter invasin constructs which contain only the adhesin domains (Y.ent 198 and Y.pst197) as well as the elongated constructs with additional invasin domains (Y.ent397 and Y.pst497) were detected in roughly equal amounts on the cell surface. Therefore, the observation of the increased binding ability of the shorter derivatives does not rely on different expression levels of the constructs. In contrast, the most 
abundant fusion (Y.pst497) showed the weakest binding to HEp-2 cells. However, the diminished binding of the longer fusions in general might be due to a steric interference of these additional domains with the binding to the integrin receptor. Among both shortened invasin fusions, the binding mediated by Y.ent198 was considerably increased in comparison to Y.pst197. These results were also confirmed using an in vitro $\mathrm{M}$ cell model to simulate the conditions in the intestinal tract (Fig. 4). Here, we investigated the shortened invasin fusions as they exhibited the strongest binding to the HEp-2 cells. The yeasts were added to monolayers that were cultured either under monoculture (Caco-2) or co-culture conditions (Caco-2/Raji B cells), the latter leading to the differentiation of a subpopulation of the Caco-2 cells into M-like cells accompanied by a decrease in TEER-value and enhanced translocation efficiency of fluorescent particles [31, 32]. Furthermore the three-fold increase in binding of the control cells without invasin demonstrates that the co-culture actually causes a change in the Caco-2 monolayers. However, this improvement became even more apparent, and statistically significant, for the invasin-expressing yeast cells. As already seen in the HEp-2 system, yeast cells expressing Y.ent198 on their surface showed the strongest binding; by co-culture of Caco-2 cells with B cells, the binding of these cells was increased more than twenty times. Importantly, the recombinant cells expressing Y.ent 198 are able to target PP as well as the overlying $M$ cells also in vivo. After feeding of mice with fluorescent labeled yeast cells it was shown via fluorescence microscopy that Y.ent 198 expressing yeast were considerably more effectively taken up in PP tissue than the respective control yeast.

The results of this study confirm that the binding properties of recombinant yeast can be improved with regard to their oral administration. In particular, the expression of the integrin-binding domain of the $Y$. enterocolitica invasin significantly increased the binding to human epithelial cells in two different in vitro model systems and also in vivo. 
Our data implicate that invasin-expressing yeast cells can be used to target $\mathrm{M}$ cells specifically in vivo and lay the basics for the development of a novel yeast-based carrier for antigens or drugs. In future studies, the invasin-expressing yeast cells will be tested for their ability of delivering a model antigen to immune cells after oral application and to subsequently induce an antigen-specific immune response. 


\section{Acknowledgements}

The authors thank Isabel Martinez-Argudo and Marc Jepson for sharing the protocol for in vitro M-like cell differentiation. This work was supported by an ESCMID research grant and a grant from Saarland Staatskanzlei (LFFP 1303) to FB. 


\section{References}

[1] M.R. Neutra, P.A. Kozlowski, Mucosal vaccines: the promise and the challenge, Nature reviews. Immunology, 6 (2006) 148-158.

[2] K.A. Woodrow, K.M. Bennett, D.D. Lo, Mucosal vaccine design and delivery, Annual review of biomedical engineering, 14 (2012) 17-46.

[3] A. Gebert, The role of $M$ cells in the protection of mucosal membranes, Histochem Cell Biol, 108 (1997) 455-470.

[4] S.A. Kerneis, A. Bogdanova, J.-P. Kraehenbuhl, E. Pringault, Conversion by Peyer's patches lymphocytes of human enterocytes into M cells that transport bacteria, Science, 277 (1997) 949-952.

[5] Y. Fujkuyama, D. Tokuhara, K. Kataoka, R.S. Gilbert, J.R. McGhee, Y. Yuki, H. Kiyono, K. Fujihashi, Novel vaccine development strategies for inducing mucosal immunity, Expert Rev Vaccines, 11 (2012) 367-379.

[6] P. Dersch, R.R. Isberg, A region of the Yersinia pseudotuberculosis invasin protein enhances integrin-mediated uptake into mammalian cells and promotes self-association, EMBO J, 18 (1999) 1199-1213.

[7] E.S. Krukonis, R.R. Isberg, Integrin beta1-chain residues involved in substrate recognition and specificity of binding to invasin, Cell Microbiol, 2 (2000) 219-230.

[8] R.R. Isberg, D.L. Voorhis, S. Falkow, Identification of invasin: a protein that allows enteric bacteria to penetrate cultured mammalian cells, Cell, 50 (1987) 769-778.

[9] J.M. Leong, R.S. Fournier, R.R. Isberg, Identification of the integrin binding domain of the Yersinia pseudotuberculosis invasin protein, EMBO J, 9 (1990) 1979-1989.

[10] A. Marra, R.R. Isberg, Invasin-dependent and invasin-independent pathways for translocation of Yersinia pseudotuberculosis across the Peyer's patch intestinal epithelium, Infect Immun, 65 (1997) 3412-3421. 
[11] V.B. Young, S. Falkow, G.K. Schoolnik, The invasin protein of Yersinia enterocolitica: internalization of invasin-bearing bacteria by eukaryotic cells is associated with reorganization of the cytoskeleton, J Cell Biol, 116 (1992) 197-207.

[12] R. Schulte, S. Kerneis, S. Klinke, H. Bartels, S. Preger, J.P. Kraehenbuhl, E. Pringault, I.B. Autenrieth, Translocation of Yersinia entrocolitica across reconstituted intestinal epithelial monolayers is triggered by Yersinia invasin binding to beta1 integrins apically expressed on Mlike cells, Cell Microbiol, 2 (2000) 173-185.

[13] J.C. Pepe, V.L. Miller, Yersinia enterocolitica invasin: a primary role in the initiation of infection, Proc Natl Acad Sci U S A, 90 (1993) 6473-6477.

[14] R.J. Critchley-Thorne, A.J. Stagg, G. Vassaux, Recombinant Escherichia coli expressing invasin targets the Peyer's patches: the basis for a bacterial formulation for oral vaccination, Molecular therapy : the journal of the American Society of Gene Therapy, 14 (2006) 183-191. [15] M. Simonet, N. Fortineau, J.L. Beretti, P. Berche, Immunization with live aroA recombinant Salmonella typhimurium producing invasin inhibits intestinal translocation of Yersinia pseudotuberculosis, Infect Immun, 62 (1994) 863-867.

[16] E. Haltner, J.H. Easson, C.-M. Lehr, Lectins and bacterial invasion factors for controlling endo- and transcytosis og bioadhesive drug carrier systems, Eur J Pharm, 44 (1997) 3-13.

[17] A.C. Stubbs, K.S. Martin, C. Coeshott, S.V. Skaates, D.R. Kuritzkes, D. Bellgrau, A. Franzusoff, R.C. Duke, C.C. Wilson, Whole recombinant yeast vaccine activates dendritic cells and elicits protective cell-mediated immunity, Nat Med, 7 (2001) 625-629.

[18] F. Breinig, T. Heintel, A. Schumacher, A. Meyerhans, M.J. Schmitt, Specific activation of CMV-primed human T lymphocytes by cytomegalovirus pp65 expressed in fission yeast, FEMS Immunol Med Microbiol, 38 (2003) 231-239.

[19] A. Ardiani, J.P. Higgins, J.W. Hodge, Vaccines based on whole recombinant Saccharomyces cerevisiae cells, FEMS Yeast Res, 10 (2010) 1060-1069. 
[20] S.B. Bazan, T. Breinig, M.J. Schmitt, F. Breinig, Heat treatment improves antigen-specific $\mathrm{T}$ cell activation after protein delivery by several but not all yeast genera, Vaccine, 32 (2014) $2591-2598$

[21] S.B. Bazan, G. Geginat, T. Breinig, M.J. Schmitt, F. Breinig, Uptake of various yeast genera by antigen-presenting cells and influence of subcellular antigen localization on the activation of ovalbumin-specific CD8 T lymphocytes, Vaccine, 29 (2011) 8165-8173.

[22] B. Walch, T. Breinig, G. Geginat, M.J. Schmitt, F. Breinig, Yeast-based protein delivery to mammalian phagocytic cells is increased by coexpression of bacterial listeriolysin, Microbes Infect, 13 (2011) 908-913.

[23] F. Breinig, T. Breinig, M.J. Schmitt, mRNA delivery to human dendritic cells by recombinant yeast and activation of antigen-specific memory $\mathrm{T}$ cells, Meth Mol Biol, 969 (2013) 163-184.

[24] B. Walch, T. Breinig, M.J. Schmitt, F. Breinig, Delivery of functional DNA and messenger RNA to mammalian phagocytic cells by recombinant yeast, Gene Ther, 19 (2012) 237-245.

[25] M.G. Kiflmariam, H. Yang, Z. Zhang, Gene delivery to dendritic cells by orally administered recombinant Saccharomyces cerevisiae in mice, Vaccine, 31 (2013) 1360-1363. [26] L. Zhang, T. Zhang, L. Wang, S. Shao, Z. Chen, Z. Zhang, In vivo targeted delivery of CD40 shRNA to mouse intestinal dendritic cells by oral administration of recombinant Sacchromyces cerevisiae, Gene Ther, 21 (2014) 709-714.

[27] B. Walch-Rückheim, M.J. Schmitt, F. Breinig, Schizosaccharomyces pombe: a novel transport vehicle of functional DNA and mRNA into mammalian antigen-presenting cells, Vaccine, 32 (2014) 6029-6033.

[28] B. Walch-Rückheim, R. Kiefer, G. Geginat, M.J. Schmitt, F. Breinig, Coexpression of human perforin improves yeast-mediated delivery of DNA and mRNA to mammalian antigenpresenting cells, Gene Ther, (2015) doi: 10.1038/gt.2015.77. 
[29] J. Sambrook, E.F. Fritsch, T. Maniatis, Molecular cloning: a laboratory manual, Cold Spring Harbor Laboratory Press, New York, 1989.

[30] H. Ito, Y. Fukuda, K. Murata, A. Kimura, Transformation of intact yeast cells treated with alkali cations, J Bacteriol, 153 (1983) 163-168.

[31] I. Martinez-Argudo, M.A. Jepson, Salmonella translocates across an in vitro M cell model independently of SPI-1 and SPI-2, Microbiology, 154 (2008) 3887-3894.

[32] I. Martinez-Argudo, M.A. Jepson, Identification of adhesin-receptor interactions driving bacterial translocation through M cells, Fut microbiol, 5 (2010) 549-553.

[33] E.T. Boder, K.D. Wittrup, Yeast surface display for screening combinatorial polypeptide libraries, Nat Biotechnol, 15 (1997) 553-557.

[34] Z.A. Hamburger, M.S. Brown, R.R. Isberg, P.J. Bjorkman, Crystal structure of invasin: a bacterial integrin-binding protein, Science, 286 (1999) 291-295.

[35] K. Moelleken, J.H. Hegemann, The Chlamydia outer membrane protein OmcB is required for adhesion and exhibits biovar-specific differences in glycosaminoglycan binding, Mol Microbiol, 67 (2008) 403-419.

[36] R. Beier, A. Gebert, Kinetics of particle uptake in the domes of Peyer's patches, Am J Physiol, 275 (1998) G130-137.

[37] D.J. Brayden, Oral vaccination in man using antigens in particles: current status, Eur J Pharm Sci, 14 (2001) 183-189.

[38] D.J. Brayden, A.W. Baird, Microparticle vaccine approaches to stimulate mucosal immunisation, Microbes Infect, 3 (2001) 867-876.

[39] M.A. Jepson, M.A. Clark, B.H. Hirst, M cell targeting by lectins: a strategy for mucosal vaccination and drug delivery, Adv Drug Deliv rev, 56 (2004) 511-525.

[40] A. Kirchhelle, N. Frühwein, D. Tobüren, Treatment of persistent diarrhea with Saccharomyces boulardii. Results of a prospective investigation, Fortschr Med, 114 (1996) 136-140. 
[41] H. Huang, G.R. Ostroff, C.K. Lee, C.A. Specht, S.M. Levitz, Characterization and optimization of the glucan particle-based vaccine platform, Clin Vac Immunol, 20 (2013) 15851591.

[42] H. Huang, G.R. Ostroff, C.K. Lee, J.P. Wang, C.A. Specht, S.M. Levitz, Distinct patterns of dendritic cell cytokine release stimulated by fungal beta-glucans and toll-like receptor agonists, Infect Immun, 77 (2009) 1774-1781.

[43] S. Chabot, J.S. Wagner, S. Farrant, M.R. Neutra, TLRs regulate the gatekeeping functions of the intestinal follicle-associated epithelium, J Immunol, 176 (2006) 4275-4283.

[44] C. Cunha, L. Romani, A. Carvalho, Cracking the Toll-like receptor code in fungal infections, Exp rev anti-infective Ther, 8 (2010) 1121-1137.

[45] T. Zhang, H. Yang, R. Wang, K. Xu, Y. Xin, G. Ren, G. Zhou, C. Zhang, L. Wang, Z. Zhang, Oral administration of myostatin-specific whole recombinant yeast Saccharomyces cerevisiae vaccine increases body weight and muscle composition in mice, Vaccine, 29 (2011) 8412-8416.

[46] R. Patterson, J. Nerren, M. Kogut, P. Court, B. Villarreal-Ramos, H.M. Seyfert, P. Dalby, D. Werling, Yeast-surface expressed BVDV E2 protein induces a Th1/Th2 response in naive T cells, Develop comp immunol, 37 (2012) 107-114.

[47] H.J. Kim, J.Y. Lee, H.A. Kang, Y. Lee, E.J. Park, H.J. Kim, Oral immunization with whole yeast producing viral capsid antigen provokes a stronger humoral immune response than purified viral capsid antigen, Lett appl microbiol, 58 (2014) 285-291.

[48] T. Heintel, F. Breinig, M.J. Schmitt, A. Meyerhans, Extensive MHC class I-restricted CD8 T lymphocyte responses against various yeast genera in humans, FEMS Immunol Med Microbiol, 39 (2003) 279-286. 
(A)

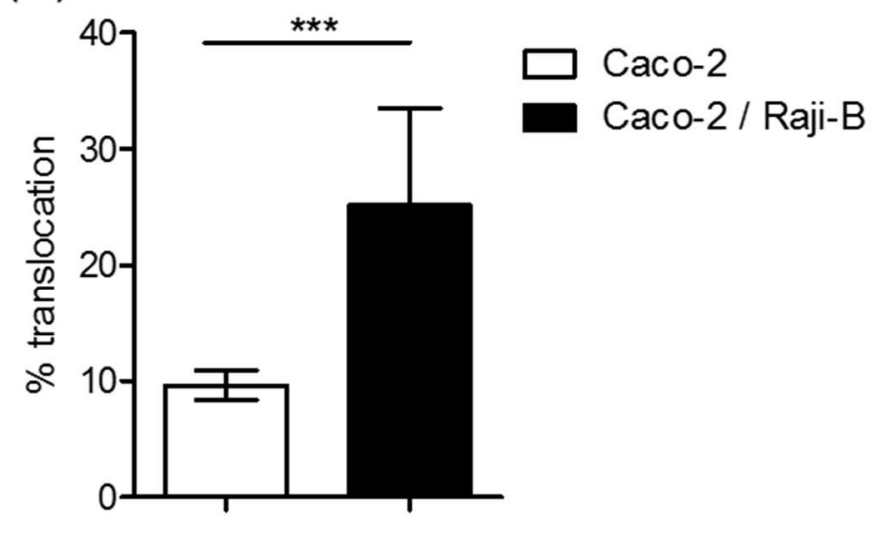

(B)

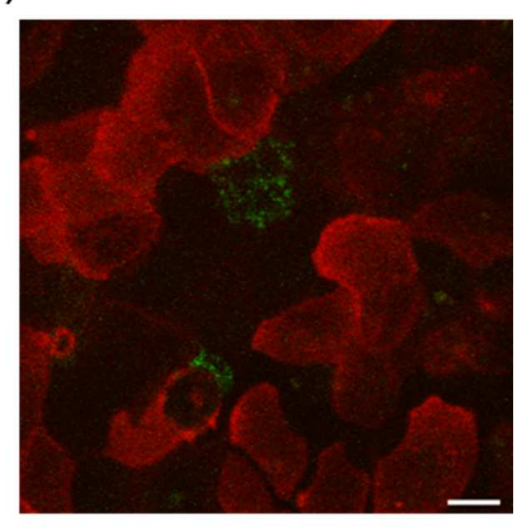

(C)

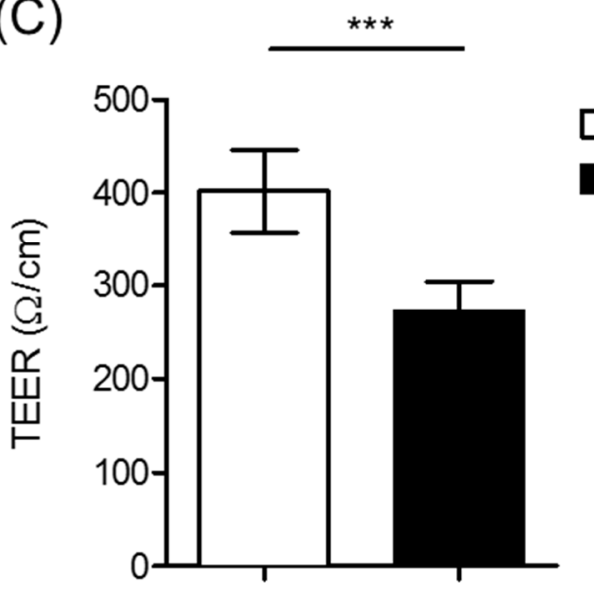

Supplementary Figure 1:

(A) Translocation efficiency of microparticles in mono- and co-culture. Cell monolayers were incubated with fluorescently labeled microparticles added to the apical chamber. Samples were taken from the basolateral chamber within 2 - 4 hours, fluorescence intensity was measured and translocation efficiency of particles was calculated ( $n=$ 10; mean \pm S.D.; Mann-Whitney test. $\left.{ }^{* *} p<0.001\right)$. (B) After 250 minutes incubation with microparticles, the Caco-2 /Raji-B monolayer was washed three times with HBSS, stained with TRITC-Phalloidin and analyzed microscopically. Red: TRITC-Phalloidin; green: microparticles; Scale bar: $10 \mu \mathrm{m}$. (C) Cell monolayer TEER values. Monolayers 
of mono- or co-cultures were rinsed with HBSS buffer and measured at $37^{\circ} \mathrm{C} . n=10$; mean \pm S.D.; t-test. ${ }^{\star * *} p<0.001$. 